Seeck, Andrea; Bär, Karl-Jürgen; Haueisen, Jens; Voss, Andreas:

Correlation between autonomic dysfunction and impaired microcirculation in patients with schizophrenia

Zuerst erschienen in: Biomedical Engineering = Biomedizinische Technik. - Berlin [u.a.] : de Gruyter. - 58 (2013), Suppl. 1, Track-G, art. [4174], 2 pp.

Erstveröffentlichung: $\quad$ 2013-09-07

ISSN (online): $\quad$ 1862-278X

ISSN (print): $\quad$ 0013-5585

DOI: $\quad 10.1515 / \mathrm{bmt}-2013-4174$

[Zuletzt gesehen: 2019-07-18]

„Im Rahmen der hochschulweiten Open-Access-Strategie für die Zweitveröffentlichung identifiziert durch die Universitätsbibliothek IImenau."

"Within the academic Open Access Strategy identified for deposition by Ilmenau University Library."

„Dieser Beitrag ist mit Zustimmung des Rechteinhabers aufgrund einer (DFGgeförderten) Allianz- bzw. Nationallizenz frei zugänglich."

"This publication is with permission of the rights owner freely accessible due to an Alliance licence and a national licence (funded by the DFG, German

DFG

Research Foundation) respectively." 


\title{
Correlation between autonomic dysfunction and impaired microcirculation in patients with schizophrenia
}

\author{
Seeck, $\mathrm{A}^{1}$, Bär, $\mathrm{KJ}^{2}$, Haueisen, $\mathrm{J}^{3}$, Voss, $\mathrm{A}^{1}$ \\ ${ }^{1}$ Department of Medical Engineering and Biotechnology, University of Applied Sciences, Jena, Germany \\ ${ }^{2}$ Department of Psychiatry and Psychotherapy, University Hospital, Jena, Germany \\ ${ }^{3}$ Institute of Biomedical Engineering and Informatics, Technical University of Ilmenau, Germany
}

andreas.voss@fh-jena.de

\begin{abstract}
Patients suffering from schizophrenia have an increased mortality risk due to cardiovascular events that might be associated with cardiac autonomic dysfunction. The aim of this study was to analyse the interdependencies between indices of autonomic regulation from heart rate and blood pressure variability, and spectral indices of Laser-Doppler-Flowmetry signals, reflecting the condition of the microcirculatory system. Therefore, we compared the correlation between indices in controls with indices in schizophrenic patients. We found that short term interaction between autonomic regulation and microcirculation decreases in schizophrenic patients compared to healthy controls while the permanently increased heart rate in patients is highly correlated with a periphery endothelial and sympathetic activation.
\end{abstract}

Keywords: Laser Doppler Flowmetry, Spectral Analysis, Heart Rate Variability, Blood Pressure Regulation

\section{Introduction}

Several studies have documented an increased mortality risk due to cardiovascular events in patients suffering from schizophrenia $[1,2]$. Some authors suggested that these events might be associated with cardiac autonomic dysfunction, specifically a decrease in vagal modulation and an increase in sympathetic activity. Bär et al. [3, 4] found a significant reduction of baroreflex sensitivity and heart rate variability as well as an increase of QT variability in schizophrenic patients indicating an autonomic imbalance.

Recently the analysis of peripheral microcirculation by means of Laser Doppler Flowmetry (LDF) has revealed a significantly altered microcirculation in patients with acute schizophrenia compared to healthy subjects [5]. The LDF is a non-invasive technology that facilitates the study of microcirculation via spectral analysis of the LDF signal. Five relevant frequency subintervals were introduced for spectral analysis of blood flow signals [6, 7]. Three of these intervals are associated with the local mechanisms of vasomotion, namely: the frequency band from $0.01-0.02 \mathrm{~Hz}$ (vascular endothelial activity), the frequency band from $0.02-0.06 \mathrm{~Hz}$ (neurogenic activity) and the frequency band from $0.06-0.2 \mathrm{~Hz}$ (myogenic response). The other two bands are related to the transmission of global hemodynamic alterations to microcirculation: from $0.2-0.6 \mathrm{~Hz}$ (respiratory activity) as well as from $0.6-1.6 \mathrm{~Hz}$ (cardiac activity). The aim of this study was to analyse the interdependencies between indices for the assessment of autonomic regulation, especially linear indices from heart rate and blood pressure variability, and indices from spectral analysis of LDF signals that reflect the condition of the microcirculatory system. Therefore, we compared the correlation coefficients between indices in healthy controls with indices in patients suffering from acute schizophrenia in order to figure out if pathologic alterations are detectable and if the interaction between autonomic regulation and microcirculatory regulation is impaired in schizophrenic patients.

\section{Methods}

In this study 37 healthy subjects (CON, mean age $34.6 \pm 11.8$ years, 21 males and 16 females) and 32 patients (PAT, mean age $35.9 \pm 11.9$ years, 16 males and 16 females) were enrolled. The investigation conforms to the principles outlined in the Declaration of Helsinki. Local ethics committee approval and the informed consent of all subjects were provided.

The LDF signals in two tissue depths (D1: 2mm; D2: $6 \mathrm{~mm}$ ) as well as an electrocardiogram (sampling frequency $1600 \mathrm{~Hz}$ ) and continuous non-invasive blood pressure (Portapres, TNO Biomedical Instrumentation) were recorded simultaneously. A post-occlusive reactive hyperaemia test was performed, i.e. a forearm ischemia was produced by a pneumatic cuff placed on the upper arm and inflated to $30 \mathrm{mmHg}$ above systolic blood pressure. After 3 minutes the cuff was deflated and registration of hyperaemia started.

From ECG and blood pressure recordings, time series of beat-to-beat intervals (BBI), systolic blood pressure (SBP) values and diastolic blood pressure (DBP) values were extracted. From the BBI time series several indices from time and frequency domain were calculated according to the guidelines of the Task Force [8]. Additionally, comparable time domain parameters were extracted from SBP and DBP time series [9].

From the two LDF signals the Short Time Fourier Transform (STFT) was calculated and features in the three frequency subintervals reflecting local mechanisms were determined referring to endothelial, sympathetic and myogenic activity. The activities of the respiratory and cardiac subintervals were not included in this analysis since they refer to global hemodynamic processes. 
Table 1: Significant correlations between indices of cardiovascular regulation and indices of local microcirculation

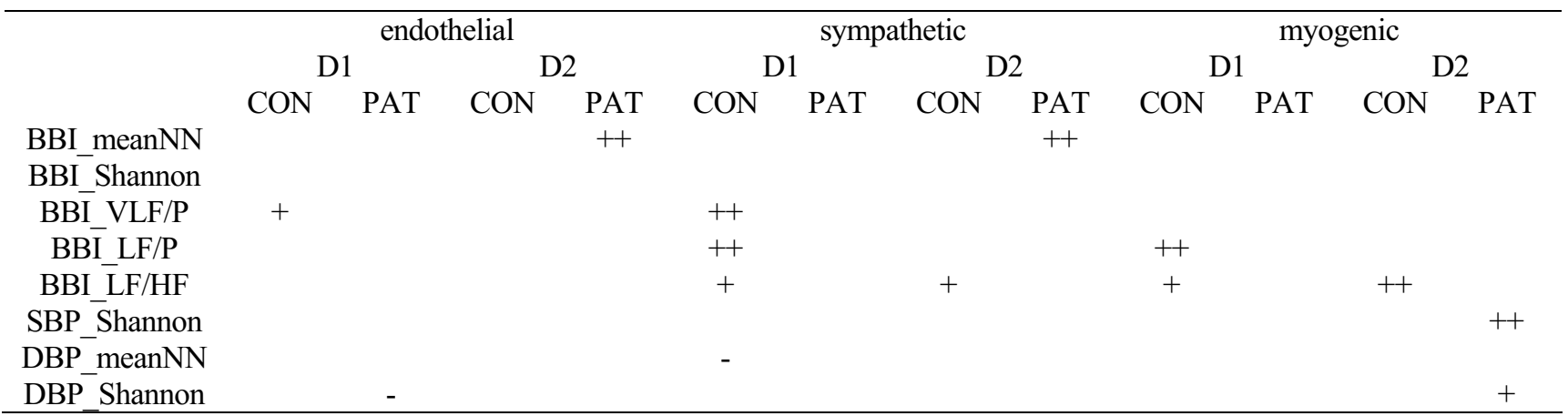

+ : positive correlation with $|\mathrm{r}|<0.4$; ++: positive correlation with $|\mathrm{r}|>0.4 ;$-: negative correlation with $|\mathrm{r}|<0.4$

A Spearmen rank correlation was performed between all indices for both groups respectively. The statistical significance level was set to $\alpha<0.05$.

\section{Results}

The analysis revealed significant correlations between indices from linear HRV and spectral indices for all three subintervals in CON. The significant correlations are presented in Table 1. The endothelial end sympathetic components in D1 showed positive linear correlations with the index VLF/P (0.003-0.04 Hz) from BBI. Additionally, the sympathetic and myogenic activities are linearly related to the frequency parameter LF/P (0.04$0.15 \mathrm{~Hz}$ ) in $\mathrm{D} 1$ and the ratio LF/HF in both tissue depths. These correlations are not significant for PAT. In contrast, PAT showed high correlations between meanNN and the endothelial and sympathetic activity in D2.

The Shannon entropies of SBP and DBP were positively related to the myogenic component in the patients group in D2. Furthermore, a negative linear relation between the mean of DBP and sympathetic activity in CON as well as between the Shannon entropy of DBP and the endothelial component in PAT were detected.

\section{Discussion}

The results of the control group show significant correlations between the central frequency parameters of $\mathrm{BBI}$ and their respective peripheral frequency intervals of microcirculation. Further on, a physiologic negative correlation between DPB meanNN and sympathetic activity is found confirming known issues [10]. In the patient group these correlations disappeared completely, probably due to the impairment of the autonomic regulatory processes. Schizophrenic patients are characterized by a strong increase of heart rate and sympathetic activity. This is accompanied by a strong positive correlation between meanNN and the endothelial and sympathetic activity in this group. The patients additionally showed an increase in the Shannon entropy of SBP and DBP ( $p=0.02$ from Mann-Whitney-U-test) compared to CON. The correlation of the myogenic component with these parameters might reflect a stronger local response to the variability of blood pressure. In conclusion the short term interaction between autonomic regulation and microcirculation decreases in schizophrenic patients compared to healthy controls while the permanently increased heart rate is highly correlated with the periphery (increased) sympathetic activation.

\section{Acknowledgement}

This study has partly been supported by grants of the University of Applied Sciences, Jena and the Deutsche Forschungsgemeinschaft (DFG: Vo 505/8-2).

\section{Bibliography}

[1] Enger, C., Weatherby, L. et. al.: Serious cardiovascular events and mortality among patients with schizophrenia, J Nerv Ment Dis, vol. 192, no. 1, pp. 19-27, Jan, 2004.

[2] Brown, S., Inskip, H. et. al.: Causes of the excess mortality of schizophrenia, $B r J$ Psychiatry, vol. 177, pp. 212-7, Sep, 2000.

[3] Bär, K.J., Koschke, M. et. al.: Acute psychosis leads to increased QT variability in patients suffering from schizophrenia, Schizophr Res, vol. 95, no. 1-3, pp. 11523, Sep, 2007.

[4] Bär, K.J., Boettger, M.K. et. al.: Decreased baroreflex sensitivity in acute schizophrenia, J Appl Physiol, vol. 102, no. 3, pp. 1051-6, Mar, 2007.

[5] Seeck, A., Israel, A.K. et. al.: Dynamic microvascular blood flow analysis during post-occlusive reactive hyperemia test in patients with schizophrenia, Ann Biomed Eng, vol. 39, no. 7, pp. 1972-83, Jul, 2011.

[6] Stefanovska, A., Bračič, M. et. al.: Wavelet analysis of oscillations in the peripheral blood circulation measured by laser Doppler technique, IEEE Trans Biomed Eng, vol. 46, pp. 1230-1239, 1999.

[7] Bracic, M., Stefanovska, A.: Wavelet-based analysis of human blood-flow dynamics, Bull Math Biol, 60, no. 5, pp. 919-35, Sep, 1998.

[8] Heart rate variability. Standards of measurement, physiological interpretation, and clinical use. Task Force of the European Society of Cardiology and the North American Society of Pacing and Electrophysiology, Eur Heart J.; vol.17, no. 5, pp. 354-81, Mar, 1996

[9] Bär, K.J., Berger, S. et. al.: Autonomic dysfunction in unaffected first-degree relatives of patients suffering from schizophrenia, Schizophr Bull, vol. 36, no. 5, pp. 1050-8, Sep, 2010.

[10] H. Suzuki, and T. Saruta, Kidney and Blood Pressure Regulation: Karger, 2004. 